\title{
MODELACIÓN DINÁMICA DE BIENES Y SERVICIOS ECOSISTÉMICOS EN LA RESERVA FORESTAL PRODUCTORA THOMAS VAN DER HAMMEN
}

\author{
Dynamic modeling of ecosystem goods and services \\ in the Thomas van der Hammen Forest Reserve
}

\author{
David Mauricio Gómez Rodríguez ${ }^{1}$ y Julio Eduardo Beltrán Vargas ${ }^{2}$
}

Gómez-Rodríguez, D. M. y Beltrán-Vargas, J. E. (2018). Modelación dinámica de bienes y servicios ecosistémicos en la reserva forestal productora Thomas van der Hammen. Colombia Forestal, 21(2), 188-204

Recepción: 9 de noviembre de 2017

\section{Resumen}

El propósito de este estudio fue realizar una modelación dinámica de la oferta y demanda de bienes y servicios ecosistémicos en relación con el cambio de uso del suelo por expansión urbana, en la reserva forestal productora Thomas van der Hammen, en la ciudad de Bogotá, Colombia. Se analizó el crecimiento poblacional, cambio de uso del suelo y de bienes y servicios ecosistémicos. La modelación dinámica se desarrolló utilizando el programa Vensim PLE®. Como resultados, se observó que para el año 2060 la reserva presentaría pérdida, cambio o deterioro en $68.75 \%$ del área que oferta bienes y servicios ecosistémicos de mantenerse las tasas actuales de cambio de uso del suelo. Además, se duplicaría la población al interior de la reserva para el periodo 2055-2056 y se perdería el agua sub-superficial como bien y servicio de provisión. Se discutió la relación existente entre la expansión urbana y el efecto negativo expresado en el deterioro de bienes y servicios ecosistémicos.

Palabras clave: áreas protegidas, bienes y servicios ecosistémicos, modelación, reserva forestal.
Aprobación: 22 de marzo de 2018

\begin{abstract}
The purpose of this study was to perform a dynamic modeling of demand and offer of ecosystem goods and services related to the land use change due to urban expansion in the Thomas van der Hammen reserve, in Bogotá, Colombia. Population growth, land use and impact on ecosystem goods and services changes were analyzed. The VensimPLE® software was used to develop the dynamic modeling. As results, it was noted that by 2060 the reserve will show loss, change or deterioration in $68.75 \%$ of the area that offers ecosystem goods and services if the actual change of land use rates is maintained. Furthermore, the population inside the reserve will be duplicated by period 2055-2056 with a possible loss of sub-superficial water used as ecosystem provision good and service. The relation between the city growth and the ecosystem goods and services deterioration was discussed.
\end{abstract}

Key words: protected areas, ecosystem goods and services, modeling, forest reserve.

Universidad Distrital Francisco José de Caldas. Bogotá, Colombia. dmgomezr@correo.udistrital.edu.co. Autor para correspondencia.

2 Universidad Distrital Francisco José de Caldas. Bogotá, Colombia. jebeltran@udistrital.edu.co 


\section{INTRODUCCIÓN}

El deterioro de los bienes y servicios ecosistémicos se puede relacionar con el crecimiento de las ciudades que carecen de modelos de ocupación regulados y coherentes con la funcionalidad ecosistémica. Ocupaciones que transforman la estructura ecológica principal(EEP) de los territorios y afectan la sustentabilidad de los mismos (Montero y García, 2017). Esto en concordancia con lo afirmado por Quintero, Castro, Garcés y Escobar (2017), quienes indican que la pérdida de la biodiversidad y el deterioro de los bienes y servicios ecosistémicos contribuyen directa o indirectamente al detrimento de aspectos del bienestar humano como la salud y la seguridad alimentaria, incrementado la vulnerabilidad y riesgo de las ciudades.

Un caso de estas transformaciones se presenta en la reserva Thomas van der Hammen (RTvdH), la cual limita con el área urbana norte de la ciudad de Bogotá y se debate entre su intervención acelerada por la urbanización o su consolidación como corredor ecológico. Teniendo en cuenta que, según la Corporación Autónoma regional de Cundinamarca (CAR, 2014b), la reserva cuenta con unas particularidades: una heterogeneidad ecosistémica con humedales y la única área representante del bosque medio denso subhúmedo secundario en planicie aluvial del río Bogotá (bosque de Las Mercedes). Zona en la que los suelos de origen aluvial dan cuenta del alto potencial productivo en términos agrícolas, pecuarios y forestales, ya que el $76.5 \%$ de los suelos son catalogados como categoría agrológica II y III con una calidad fundamental para la seguridad alimentaria y el potencial establecimiento de proyectos productivos tanto agroforestales como silvopastoriles con un enfoque ecológico.

En oposición a lo anterior, esta área puede ser transformada por la expansión urbana, razón por la cual se estructuró un sistema simplificado basado en la modelación dinámica y la simulación, ya que son herramientas útiles para describir, analizar y evaluar sistemas. Según Villanueva-Solis (2013), estas herramientas han sido utilizadas en diferentes disciplinas y áreas del conocimiento con el fin de entender sistemas en los que se concibe cualquier aspecto del mundo como la interacción causal entre atributos que lo describen y, que según Ibarra (2013), se construyen como representaciones sistémicas con flechas y puntos, denominadas diagramas causales.

Teniendo en cuenta lo anterior, un modelador establece hipótesis y relaciones que posteriormente se visualizan en un diagrama de niveles y flujos, en el que se realiza una cuantificación del diagrama causal para, de este modo, obtener un conjunto de ecuaciones que permitan ver al tomador de decisión el comportamiento del sistema de interés a través de simulaciones en un software especializado.

Como establecen Mas y Flamenco (2011), existen diferentes modelos de cambio de cobertura y uso del suelo que han sido desarrollados para satisfacer las necesidades de gestión de la tierra y otros elementos ecosistémicos. Estos buscan predecir para entender, evaluar y proyectar el papel de los cambios en el funcionamiento del sistema terrestre.

Es así como los procesos de modelación aportan a la generación de metodologías y herramientas, ya que se basan, como indican Mas y Flamenco (2011), en el análisis de los cambios pasados que permiten evaluar las tasas de cambio entre los diferentes tipos de coberturas y uso del suelo, teniendo en cuenta la localización y las variables que influyen la distribución de los cambios. Lo cual permite predecir escenarios para la toma de decisiones para mejorar la calidad de vida y gestión sustentable de los ecosistemas y territorios.

Existen diferentes paquetes de modelación que tienen funciones y herramientas con requisitos y supuestos distintos, ya que emplean distintos tipos de algoritmos que pueden ser más o menos adecuados dependiendo de los datos de entrada y el propósito de la modelación. Según Ibarra (2013), con el desarrollo de la modelación dinámica se generan sistemas de información que poseen soporte de decisiones con una disminución de los 
niveles de incertidumbre para que, por tanto, las alternativas que se desarrollen tengan umbrales de riesgo más bajos.

El objeto del estudio fue estimar el estado de los bienes y servicios ambientales en la RTvdH en relación con las áreas y coberturas vinculadas al flujo de los bienes y servicios ambientales; además de predecir posibles comportamientos en las variables población, recurso hídrico y suelo.

\section{MATERIALES Y MÉTODOS}

\section{Área de estudio}

La RTvdH tiene 1395.16 ha y está localizada al noroccidente de la ciudad de Bogotá, en las localidades de Suba y Usaquén, en la sabana de Bogotá, centro geográfico de Colombia, sobre el flanco occidental de la cordillera Oriental, parte sur del altiplano cundiboyacense, entre los 2550 y 2560 m de altitud.

Este territorio, según Gómez (2017), ha crecido de forma ilegal, desordenada y con carencia de un modelo de crecimiento urbano, lo cual ha llevado al deterioro paulatino de la estructura ecológica principal. Esto coincide con lo que establecen Barroso, de Zárate y Álvarez (2010), como una ciudad que se extiende por el territorio devorando suelo, consumiendo energía, agua y materiales; acrecentando sus problemas de circulación y, a su vez, emitiendo contaminantes.

La ocupación sistemática del espacio supone la desvaloración de los espacios agrícolas, forestales y naturales; aplicando una lógica sectorial basada en la movilidad privada. Esto explica claramente la degradación ambiental en la $\mathrm{RTvdH}$, parte del hábitat de una amplia biodiversidad, representada especialmente en aves nativas y migratorias, mamíferos y especies de flora. Lo anterior, según Casas y Porras (2017), ya que el principal uso actual del suelo en la reserva es agropecuario, el cual se extiende sobre el $59.28 \%$ de la misma, seguido del cultivo de flores (11.37\%). Asimismo, ningún uso urbano se extiende en mayor área que los anteriores, pero en conjunto ocupan el $15.43 \%$ de la reserva, y el uso dotacional educativo es el urbano con mayor extensión en la reserva (4.61\%). Por otra parte, el uso residencial se duplicó en los dos últimos años, pasando de $0.84 \%$ al $1.56 \%$ (CAR, 2014a).

La CAR (2014a) identifica la formación sabana en casi un $80 \%$ de la zona de estudio, la cual se encuentra expuesta en nivel superficial por lo que cuenta con niveles freáticos y no piezométricos, lo que hace que tenga característica de acuífero libre. Inclusive, la existencia de acuíferos libres es bastante importante y se lleva a cabo hasta una profundidad de 7 metros, mientras que la existencia de acuíferos semiconfinados es menor, encontrándose a partir de los $30 \mathrm{~m}$ de profundidad. Es así como la zona de estudio incluye dos áreas de drenaje que corresponden al humedal de Torca-Guaymaral, en el nororiente (cuenca Torca) y el humedal de La Conejera (subcuenca del mismo nombre) en el noroccidente; ambos drenajes tributan al río Bogotá. La conectividad hídrica de las aguas superficiales está representada en tres ejes principales, el canal de Torca-Guaymaral, los vaIlados paralelos a la vía Cota-Suba y la quebrada La Salitrosa. Estos hacen parte de ecosistemas naturales de gran importancia ecológica por las funciones ambientales que cumplen, siendo áreas estratégicas para la conectividad entre corredores ecológicos y la estructura ecológica regional que aportan al equilibrio ambiental de la ciudad y la región (Sánchez Díaz, 2016).

Gómez (2017) observó que en la RTvdH se reconocen en mayor proporción los bienes y servicios ecosistémicos (BySE) de regulación (54\%), seguido de los BySE de provisión (29\%) y por último los bienes BySE culturales (17\%). Como los bienes y servicios de regulación más relevantes en el área. se resalta que, a pesar de ser un área altamente intervenida, la población reconoce la conservación de especies y el mantenimiento de la calidad del aire, seguido de la conservación del bosque y las áreas de recarga de acuíferos. 
Con relación a los bienes y servicios de provisión, se observa que los que se perciben como de mayor importancia son el agua subterránea y el suelo, seguidos del agua superficial, los recursos genéticos como semillas y los alimentos.

Finalmente, respecto a los bienes y servicios culturales se identificó que, debido a la influencia de los centros deportivos, la recreación con el $31 \%$ tiene gran importancia en los servicios de la reserva, y en un segundo plano, los bienes y servicios correspondientes a la belleza escénica y los enriquecimientos espirituales, y con un menor porcentaje, la inspiración artística y la investigación a pesar de los más de 50 estudios que se reportan en la RTvdH.

\section{METODOLOGÍA}

La modelación dinámica de la oferta y demanda de bienes y servicios ecosistémicos con relación al cambio de uso del suelo incluyó valores de estudios realizados por la Car, la Secretaría Distrital de Planeación (SDP), el Departamento Administrativo Nacional de Estadística (Dane), entre otros.

Es así como se definieron las variables necesarias para determinar el comportamiento de algunos aspectos ambientales de la RTvdH como: el crecimiento poblacional, el cambio del uso del suelo, los cambios de las reservas de agua subterránea y la afectación de bienes y servicios ambientales de regulación, provisión y culturales.

Lo anteriormente mencionado se desarrolló utilizando como herramienta el programa VensimPLE (Ventana Systems, 2015), herramienta que Eberlein y Peterson (1992) describieron como un entorno de software interactivo que permite el desarrollo, exploración, análisis y optimización de modelos de simulación.

Así mismo, se utilizó como prueba estadística de validación la prueba de análisis de sensibilidad multivariable de Montecarlo que, según García, Hoz Sánchez y Pfaffenbichler (2008), es el método más recomendado para tratar con tendencias de falla y reparación variables. Este método requiere recopilar gran cantidad de datos para establecer las combinaciones de eventos que definen el comportamiento del sistema con estimaciones más precisas que dependen del número de experimentos simulados (Narváez, 2015).

Por último, el enfoque de simulación se basa en representar el proceso real y el comportamiento aleatorio del sistema como un diagrama de flujo, en el cual se recrean sus posibles escenarios. En general, estos métodos tratan el problema como una serie de experimentos soportados por computador y el sistema es evaluado en términos del número de veces que ocurre el evento de interés. Según Verma, Srividya y Karanki (2010), la simulación Monte Carlo es la técnica más representativa de este tipo, ya que el modelo representa y luego realiza 200 simulaciones cambiando automáticamente los parámetros.

\section{Modelo dinámico}

Por medio de la construcción del modelo se relacionaron áreas y el uso que se da a algunas relacionadas a los bienes y servicios ambientales con el fin de determinar el ritmo de deterioro de los mismos por el cambio de uso del suelo con tendencia a la urbanización y el crecimiento de la población al interior de la reserva. Es así como se construyen supuestos que ayudan a simplificar y analizar el modelo. De esta forma, se consideran como supuestos las siguientes premisas:

a. Al aumentar la población se incrementa el cambio del uso del suelo, incrementando la demanda de suelo.

b. Una mayor presión y deterioro de los bienes y servicios ecosistémicos reduce la cantidad de hectáreas disponibles para la consolidación de la reserva con un uso forestal o con coberturas naturales.

c. Los cambios de uso del suelo con tendencia a la urbanización cambian y deterioran los bienes y servicios ecosistémicos. 


\section{Arquitectura del modelo}

En el modelo se establecieron subsistemas integrados a partir de la dinámica poblacional y el cambio del uso del suelo, relacionándolo con los componentes ambientales agua y usos del suelo de la RTvdH. Estos fueron agrupados en bienes y servicios ambientales, culturales, de provisión y regulación, con el fin de identificar el cambio y deterioro de los mismos (figura 1).

El recurso hídrico se modeló en un subsistema diferente, buscando integrar al modelo la demanda de agua, las reservas de agua subterránea y el agua superficial. Ya que, según Gómez (2017), el mayor aprovisionamiento en la reserva está dada por la obtención de agua subterránea y de suelo.

Teniendo como referencia el diseño metodológico de Wei (2012), el proceso de modelación dinámica empleó los siguientes pasos: (1) búsqueda de información y la definición de objetivos de simulación; (2) determinar el alcance del sistema; (3) la construcción del diagrama causal; (4) el diseño de una estructura al identificar variables y unidades; (5) el desarrollo de diagramas de flujos; (6) supuestos del modelo y la formulación del modelo matemático; (7) la calibración y validación de modelo; y (8) la implementación del modelo.

Las variables que se han identificado en la RTvdH para estructurar el modelo son las presentes en la tabla 1.

Para la construcción del sistema dinámico se tuvieron en cuenta las áreas de infiltración para recarga de acuíferos de la reserva que, según la CAR (2014a), corresponde a la franja de 30 m localizada al pie del cerro La Conejera (borde occidental del cerro) y el costado oriental del polígono ubicado en la localidad de Usaquén contra la Reserva Forestal Protectora Bosque Oriental de Bogotá.

Tabla 1. Elementos y valores asociados al modelo

\begin{tabular}{|c|c|c|}
\hline Elemento asociado al modelo & Datos utilizados & Fuente \\
\hline \multicolumn{3}{|l|}{ Uso del suelo actual } \\
\hline Suelo de la reserva & 1395.16 ha & \\
\hline Cambio de uso del suelo periodo 2001-2014 & 267.45 ha & \\
\hline Cobertura forestal & 103.10 ha & \\
\hline Coberturas artificiales & 165.60 ha & \\
\hline Bosques & 35.7 ha & \\
\hline Agropecuario & 985.6 ha & \\
\hline Comercio y servicios & 7.43 ha & \\
\hline Dotacional & 95.36 ha & \\
\hline Conservación & 108.54 ha & \\
\hline Población & & Car (2014b) \\
\hline Población residente & 2095 habitantes & \\
\hline Población flotante & 21284 habitantes & \\
\hline Tasa de crecimiento poblacional Suba 2015 & $\begin{array}{l}\text { Exponencial } 2.36 \\
\text { Geométrico } 2.39\end{array}$ & \\
\hline Tasa de migrantes netos por mil (Suba) & 11.21 Habitantes & \\
\hline Tasa natalidad por mil & 16.51 & \\
\hline \multicolumn{3}{|l|}{ Recurso hídrico } \\
\hline Precipitación promedio & 982 mm/año & \\
\hline Agua acumulada acuíferos & $412000000 \mathrm{~m}^{3}$ & \\
\hline Agua superficial $27.87 \%$ del área. & $\begin{array}{c}278669 \mathrm{~m}^{2} \\
(388831092 \text { ha })\end{array}$ & \\
\hline $\begin{array}{l}\text { Concesiones de agua superficial y subterránea } \\
\text { para el consumo }\end{array}$ & 115.82 Lps & \\
\hline
\end{tabular}


Estas áreas ocupan 4.35 ha, equivalentes al 0.31\% de la reserva. Además, para la construcción del modelo se tuvo en cuenta el consumo de agua en los modelos productivos agropecuarios los cuales son de aproximadamente $16000 \mathrm{~m}^{3}$.ha.año ${ }^{-1}$ por cosecha (Siac, 2012); en un área en uso agropecuario que se estima en 985.60 ha. Además, se utilizaron los datos correspondientes al cambio de uso del suelo en el área de influencia que, según la Car (2014), es de 20.57 ha por año.

La estructura del modelo simplifica la realidad para analizar los bienes y servicios ambientales asociados a coberturas y usos del suelo definidos en el plan de manejo ambiental de la reserva, los cuales fueron integrados en la oferta de la siguiente manera, teniendo en cuenta que los valores de las áreas asociadas a los usos y coberturas que aportan al flujo de bienes y servicios ecosistémicos se les dieron una valoración positiva:

- Regulación: aportan a la auto-regulación de los procesos ecosistémicos. Usos vinculados; usos en coberturas forestales+, bosques + y conservación +
- Provisión: aportan a la provisión de bienes y productos que se obtienen de los ecosistemas. Usos vinculados; uso dotacional- y agropecuario+

- Culturales: aportan a la obtención de los beneficios no materiales obtenidos de los ecosistemas. Usos vinculados; uso en coberturas artificiales+, comercio y servicios-.

Los valores y formulas utilizados se encuentran en la modelación dinámica se encuentran anexos (anexo 1).

\section{RESULTADOS}

\section{Modelación}

En la figura 1 se observa lo obtenido de las relaciones que fueron el resultado del diagrama causal. Este permite establecer las relaciones existentes entre la población, el recurso hídrico y los bienes y servicios ambientales.

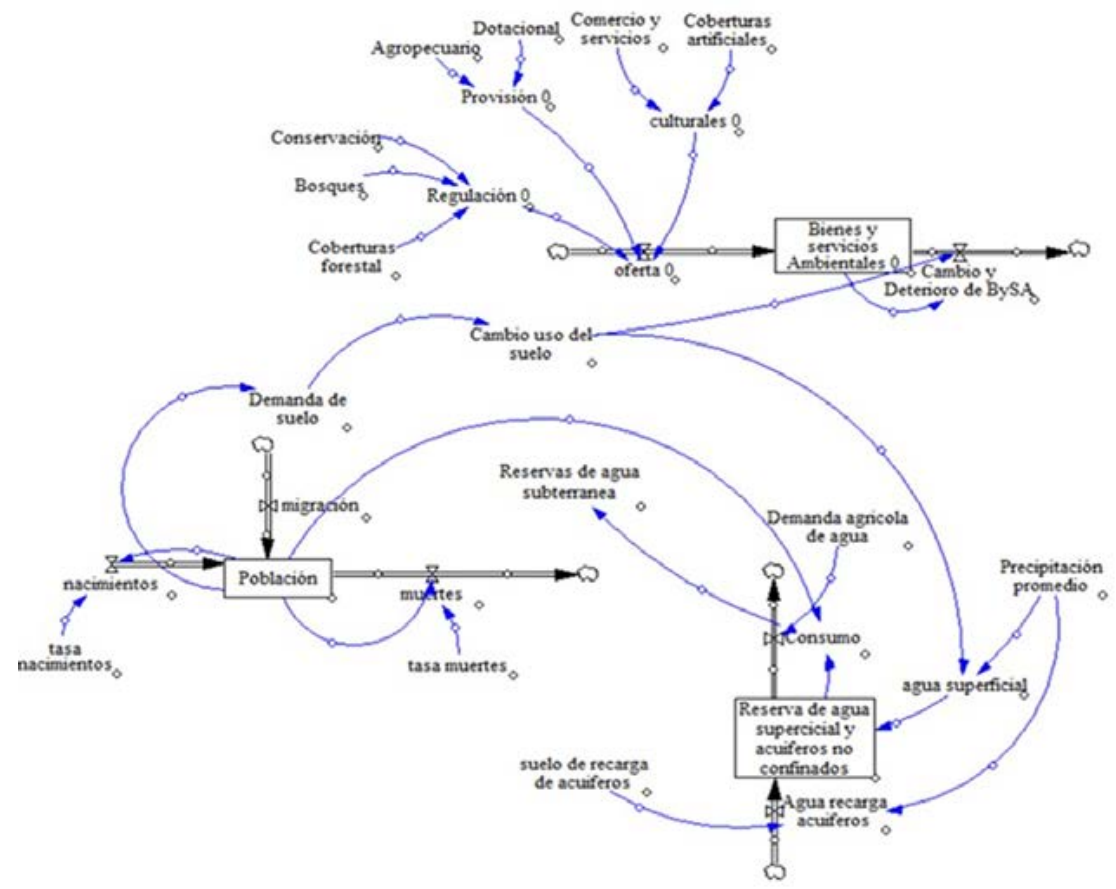

Figura 1. Estructura del modelo aplicado a la RTvdH. 
Teniendo en cuenta que algunos usos del suelo generan una presión negativa y otros una presión positiva en la oferta de bienes y servicios ambientales, se determinó que a partir de los usos actuales en la RTvdH el área del suelo que oferta bienes y servicios ecosistémicos inicialmente se estima en 1295 ha. De no ser afectadas por la demanda de suelo para urbanizar se podría incrementar esta y para el año 2041 llegaría a casi 1500 ha, que ofertarían bienes y servicios ecosistémicos. Lo anterior contrasta con la pérdida de los bienes y servicios ambientales si se alteran por el cambio de uso del suelo con tendencia a la urbanización, como se observa a continuación (figura 2):

Como se observa en la figura 2, el incremento de cambio del uso del suelo genera cambios y deterioro de BySA. El cambio y deterioro en la oferta de bienes y servicios ambientales hasta el año 2060 correspondería al 37\%, cuando la reserva presente cambios en el $68.75 \%$ del área que oferta bienes y servicios ambientales, si se mantienen las tasas actuales de cambio de uso del suelo con tendencia a la urbanización.

En la figura 3 se observa que, debido a una posible alteración de los suelos y las zonas de recarga, existe una alteración de la regulación hídrica, ya que se reduce el agua de los acuíferos no confinados, que se calculan en el 3.9\% de las reservas totales $\left(16068000 \mathrm{~m}^{3}\right.$ ), reduciendo por completo el aprovechamiento del agua de sub-superficial como bien de provisión en dos años; esto debido a la posible alteración de los suelos y las zonas
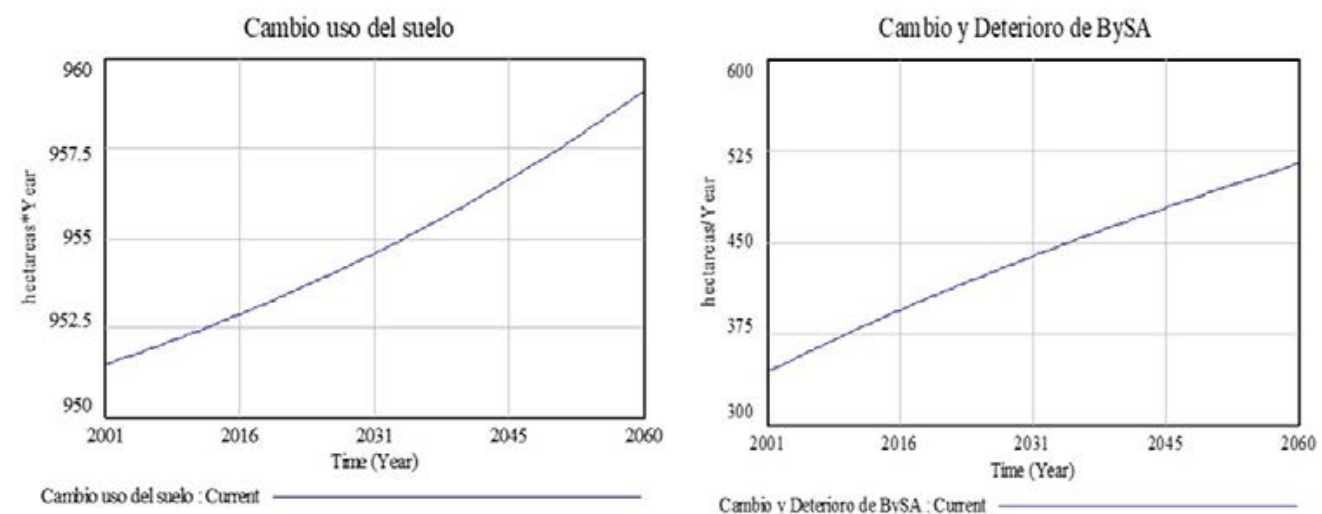

Figura 2. Modelación cambio de uso del suelo y cambio y deterioro de bienes y servicios ambientales RTvdH.

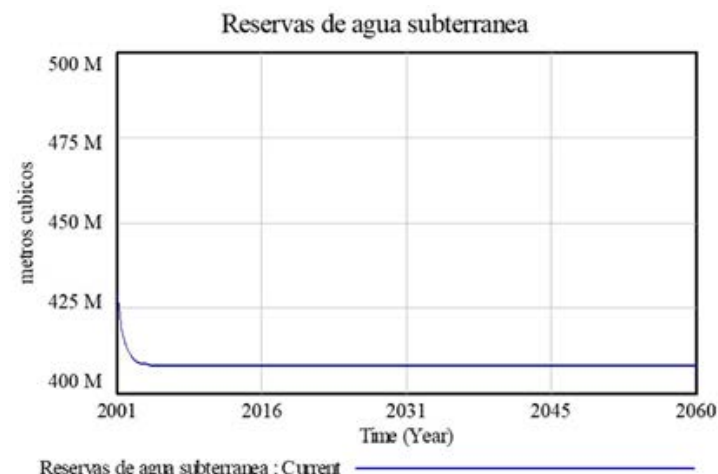

Figura 3. Modelación de reservas del recurso hídrico RTvdH. 
de recarga. En la simplificación que permite hacer el modelo, dentro de los siete primeros años se afectarían totalmente las dinámicas hídricas, incrementando la presión en un $0.98 \%$ de las aguas subterráneas de acuíferos confinados; pero manteniéndose constantes las reservas de aguas subterráneas que se encuentran a más de 30 metros de profundidad. Aparte del tiempo inicial de la modelación, esto ocurriría dependiendo de las tasas de transformación del uso del suelo en las áreas de recarga de acuíferos.

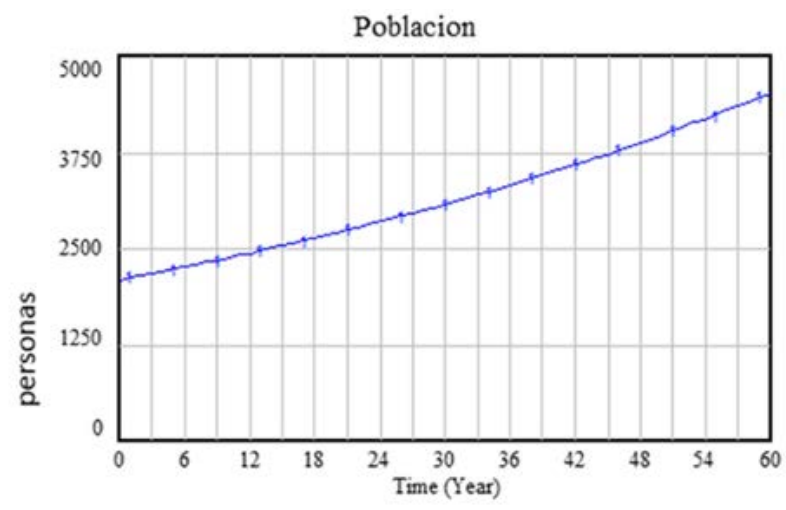

Figura 4. Modelación de la población RTvdH.

Se identificó que la población al interior del área de estudio se incrementaría (figura 4). Con referencia al censo poblacional del año 2005 (año 0), para el periodo 2055-2056 se duplicaría la población. Este resultado corresponde a la modelación de los datos de las proyecciones de las tasas de crecimiento poblacional, natalidad, mortalidad, migraciones netas, etc., basados en fuentes oficiales de la Secretaría de Planeación Distrital de Bogotá. El incremento de la población en el modelo influye directamente en las tasas de cambio de uso del suelo.

En el modelo se observó que el aumento de la población podría hacer que para el 2060 se acumule una demanda de suelo urbano de casi 15 ha en las condiciones actuales. Pero con el cambio de uso del suelo con tendencia a la urbanización se podrían transformar entre 143 y 150 ha anuales, llegando a ocupar en nueve años el suelo destinado a la consolidación de la RTvdH.

\section{Montecarlo}

La prueba de sensibilidad se realizó con la variable correspondiente a precipitación, debido a que de esta variable depende la modelación de otros procesos, ello verifica la importancia de modelar el recurso hídrico en los componentes ambientales y demuestra que los datos están muy acordes con los análisis realizados, pues no se sobreestiman ni se subestiman los datos y valores simulados. Los límites de confianza de la precipitación están dentro de los 0- y $0.00001 \mathrm{~m}^{3}$ anuales (figura 5).

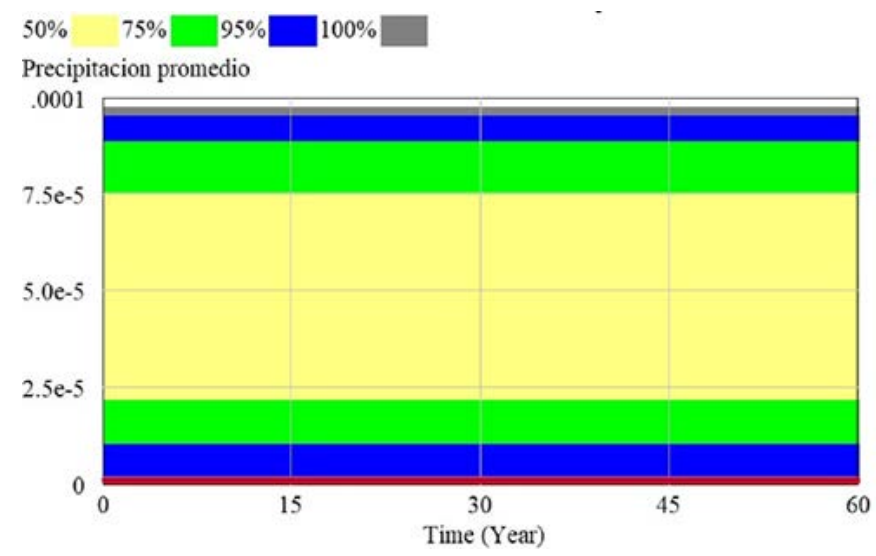

Figura 5. Resultado arrojado por la prueba de sensibilidad en Vensim PLE. 


\section{DISCUSIÓN}

Según Gómez (2017), las sociedades transforman dinámicamente su entorno y difícilmente encuentran un límite en la estructuración de las ciudades, ya que estas transformaciones provienen de visiones territoriales y necesidades divergentes que al encontrarse en un mismo espacio generan conflictos de intereses. Mazza (2017) identifica que cada vez son mayores los desequilibrios y contrastes territoriales en ciudades latinoamericanas. Estos se evidencian en conflictos ambientales por la tenencia y el uso del suelo en los que la construcción de paisajes mezcla aspectos urbano-rurales en las periferias con tipologías de vivienda disímiles, lo cual se acentúa en sociedades con grandes brechas socioeconómicas que carecen de modelos de ocupación regulados y que no tienen en cuenta la conservación de la estructura ecológica principal de los territorios la cual, según van Der Hammen y Andrade (2003), sustenta los bienes y servicios ecosistémicos.

Rojas (2016) afirma que en la actualidad Bogotá afronta transformaciones por la presión de construir viviendas en ecosistemas frágiles, como áreas rurales o zonas propensas a las inundaciones, que al mismo tiempo se deben proteger para asegurar la capacidad de resiliencia.

En concordancia con Mosquera (2015), la ocupación y el uso del suelo, las prácticas agrícolas insostenibles y la poca presencia institucional afectan los servicios ecosistémicos. En la modelación se observó que estos pueden llegar a un estado crítico, poniendo en riesgo la sustentabilidad de la RTvdH (figura 2), pues se plantean modelos de desarrollo que no tienen en cuenta las relaciones complejas que se entretejen en los socio-ecosistemas, dando prioridad a la transformación de ecosistemas para emplazar equipamiento de la ciudad, desconociendo la funcionalidad y complejidad territorial.

Además, la sociedad obtiene muchos beneficios del crecimiento económico y uso del suelo (Eppink, 2004) que, según Calvachi (2013), genera conflictos sociales como la expansión desmesurada de la ciudad. Esto se deriva en degradación ambiental o en depresión social y económica. Razón por la cual se hace énfasis en tener en cuenta la estructura ecológica de soporte definida por van der Hammen y Andrade (2003) como la expresión territorial de los ecosistemas naturales, agroecosistemas y sistemas urbanos construidos que soportan y aseguran a largo plazo los procesos, sustentan la vida humana, la biodiversidad, el suministro de servicios ambientales y la calidad de la vida. Es así como la conservación y restauración de la Estructura Ecológica Principal (EEP) son el fundamento territorial para el desarrollo verdaderamente sostenible.

Teniendo en cuenta lo anterior, y considerando la RTvdH como parte de la estructura ecológica principal, la Car (2014a) indica que la presencia de acuíferos en la zona es evidente, principalmente debido a que se encuentra ubicada dentro de la gran cuenca artesiana de la sabana de Bogotá, además de estar en el borde oriental, el cual constituye el límite geográfico de la sabana y una de las zonas de recarga más importantes para los acuíferos profundos.

Igualmente, la Car (2014b) identifica características de porosidad y permeabilidad de las rocas en este sector que producen varios niveles de almacenamiento y flujo de agua, generando dos grandes acuíferos semiconfinados y uno libre. Indiscutiblemente estos representan una buena fuente de aguas subterráneas y condicionan la existencia de humedales en la zona de estudio, razón por la cual el cambio del uso del suelo con tendencia a la urbanización estaría en contravía a los elementos reguladores, ya que generan una mayor escorrentía superficial.

Los argumentos que indican que el recurso hídrico subterráneo no es afectado por la urbanización y desconocen la importancia de los suelos allí presentes con los que se justifica la expansión urbana son desmentidos por Torres, Martínez y Nájera (2017), quienes han demostrado que en los procesos de expansión urbana se genera destrucción 
de ecosistemas, contaminación, disminución o pérdida de cuerpos de agua, sobreexplotación de mantos acuíferos y perdida de bosques. Lo anterior se refleja en el caso de los cambios de uso y cobertura de suelo por la expansión urbana en la ciudad de Viña del Mar, Chile, en la que se demostró que la disponibilidad del agua, permeabilidad de los suelos y abundancia en la vegetación han sido afectados por las áreas urbanas o caminos de acceso.

La construcción y análisis del modelo permite interpretar que con o sin una intervención de expansión urbana planificada, aun así, se están transformando los suelos y se alteran las áreas relacionadas a la oferta de los bienes y servicios ecosistémicos. En Bogotá, en promedio son urbanizadas 26207 ha.año ${ }^{-1}(0.08 \%$ del área urbana de la ciudad), en la reserva el cambio del uso del suelo en los últimos 13 años, según la Car (2014a), fue de 20.57 ha.año-1 $(1.4 \%$ del área de la reserva). Cambio de uso que está relacionado con la disposición de escombros, la construcción ilegal, la pérdida de coberturas de bosque, entre otros. Según Acevedo (2003), entre 1940 y 1965 el cambio de uso del suelo en la zona fue de 25 ha.año $^{-1}$, reduciéndolo el bosque de las mercedes a casi 6 ha. Así mismo, podemos agregar que el uso del suelo formado en conjunto por el tejido urbano discontinuo es de $1.03 \%$ y se identifica un $0.69 \%$ del área de la reserva afectada por escombros (Gómez y Jiménez, 2014).

Inclusive las áreas que ofertan bienes y servicios ecosistémicos se pueden ver afectados debido a factores como la contaminación, ya que las ciudades enfrentan una preocupante degradación ambiental con altos niveles de contaminación que resultan de los modelos de desarrollo (Montero y García, 2017). Como, por ejemplo, los modelos productivos basados en el modelo de revolución verde y el incremento en el consumo de recursos naturales y servicios públicos fruto del incremento de la población al interior de la reserva, la cual se calcula que pasará de 2095 a 5000 habitantes aproximadamente en los próximos 60 años.
El incremento de la población se tuvo en cuenta para modelar la demanda de suelo con tendencia a la urbanización, ya que el tamaño mínimo del lote unifamiliar según el Decreto 2083/2014 del Ministerio de Ambiente, Vivienda y Desarrollo Territorial es de $35 \mathrm{~m}^{2}$. Así mismo, según Acevedo (2003), el índice de elasticidad suelo-población es definido como el incremento del área desarrollada sobre el incremento de la población de 30 $\mathrm{m}^{2} \cdot \mathrm{hab}^{-1}$; datos con los cuales se justifican áreas de expansión urbana.

De la misma forma, Álvarez (2010) indica que la demanda creciente por servicios ambientales y las consecuentes transformaciones de los ecosistemas pueden exceder la habilidad de la tierra para proveerlos. Estos están asociados a las funciones de hábitat, la provisión de los ecosistemas naturales de sitios de refugio y reproducción para organismos silvestres como componentes del manejo del ambiente para un desarrollo sustentable; es así como los cambios de uso y cambios de cobertura del suelo generan cambios en los bienes y servicios ecosistémicos (fenómeno que está considerado entre las principales fuerzas que inciden sobre el cambio y deterioro global).

De esta manera, González (2017) relaciona el cambio de uso del suelo con la afectación dinámica del paisaje, especialmente en las ciudades, lo que incrementa la vulnerabilidad ante eventos meteorológicos extremos y reduce la capacidad de adaptación.

Teniendo en cuenta lo anterior, y según los resultados de la modelación, en promedio se podría generar un acelerado cambio de uso del suelo, ya que se pueden transformar anualmente entre 143 y 150 ha.año $^{-1}$, Ilegando a ocupar en 9 años el suelo destinado a la $\mathrm{RTVdH}$ y reduciendo la capacidad de la ciudad para consolidar áreas estratégicas para la adaptación a fenómenos de variabilidad y cambio climático. Como lo indicó la Academia Colombiana de Ciencias Físicas y Naturales (ACCEFY, 2011) al afirmar que esta área aportaría a la disminución de emisiones de gases de efecto invernadero, la captura y almacenamiento de carbono, a la 
conectividad y mantenimiento de los ecosistemas regionales con un modelo de protección del ambiente capaz de contribuir a la preservación de la vida, con el consecuente bienestar humano.

Es importante saber que este modelo identifica los procesos acelerados que puede alterar el área delimitada, ya que en la realidad la tenencia de la tierra corresponde a 30 empresas y sociedades que poseen 1020 ha de la zona protegida (Correa y Suárez, 2016). Es decir, el 76\% del total de la RTvdH se podría urbanizar, lo cual inicialmente reduciría el ritmo de cambio del suelo, pero dependiendo de los cambios en la política distrital podría afectarse en la totalidad la reserva. Es decir, solo entre agroindustriales y empresas interesadas en la construcción suman el 55\% de la RTvdH.

Dadas las consideraciones anteriores, en el modelo se observa que en 60 años la población al interior de la reserva se duplicaría llegando a 5000 habitantes y se alteraría la recarga de agua de los acuíferos no confinados, que se calculan en el $3.9 \%$ de las reservas totales $\left(16068000 \mathrm{~m}^{3}\right)$. Lo anterior reduciría por completo el aprovechamiento del agua sub-superficial como un bien y servicio de provisión en dos años, debido a la posible alteración de los suelos y las zonas de recarga, resaltando que la recarga principal de los acuíferos semiconfinados se da por infiltración de aguas en las zonas en las que aflora la roca y en forma secundaria por percolación de aguas en los acuíferos superiores; para el acuífero libre, la recarga se da por infiltración de aguas provenientes de precipitación y escorrentía superficial (CAR, 2014a). Lo cual se podría comprobar de una mejor forma si se realiza la modelación dinámica del recurso hídrico con relación a factores sociales y económicos que, según Kang (2012), deben ser tenidos en cuenta.

De acuerdo a lo anteriormente expuesto y lo indicado por Cortés, García y Díaz (2015), la expansión urbana relacionada con el crecimiento poblacional incrementa la demanda de bienes y servicios ecosistémicos, afectando negativamente los ecosistemas que los proveen. Esto hace que los ecosistemas pierdan su reconocimiento de capital natural dentro del sistema económico, siendo degradados debido a la producción económica y el consumo humano.

De hecho, Montero y García (2017) señalan que en América Latina y el Caribe la expansión urbana, además de su impacto ambiental, implica un importante costo económico al aumentar todos los costos de gestión de la ciudad. Es decir, la red de infraestructura (agua, electricidad, transporte, comunicación, carreteras, etc.) no solo tiene que ampliar su cobertura a zonas cada vez más alejadas, sino que a medida que va bajando la densidad de la población su costo por habitante se incrementa.

Sin embargo, es importante reconocer las limitaciones del modelo que utiliza tasas, valores y constantes que se derivan de los estudios realizados con anterioridad. La simulación relacionó la afectación de los bienes y servicios ecosistémicos como consecuencia del cambio de uso del suelo en áreas que sustentan estos mismos, pero que no son afectados de igual forma ni en igual magnitud. Aun así, es importante reconocer la utilidad de la modelación, ya que como indican Zhan, Zhang, Ma y Chen (2012), en la moderación del crecimiento urbano en la región China de Tianjin, los resultados de estas metodologías son directamente útiles para los planificadores y formuladores de políticas al comparar diferentes consecuencias dinámicas como consecuencia de las políticas y decisiones, siendo una herramienta útil para identificar que tan rápido puede ser una región o área urbanizada y como puede desarrollarse bajo principios de sostenibilidad ecológica y económica.

Así mismo, Zhan et al. (2012) resaltan la importancia de integrar los entornos ambiental-social y económico para desarrollar sistemas que ayuden a evaluar los posibles impactos de las políticas gubernamentales en desarrollo. Quintero et al. (2017) señalan la dificultad conceptual, técnica y operativa al momento de espacializar los bienes y servicios ecosistémicos.

Los resultados de esta investigación demuestran la relación existente que, según Torres et al. (2017), 
existe entre la urbanización y los daños en el ambiente en ciudades, afirmación que ha sido demostrada con diferentes metodologías y herramientas. Por ejemplo: los estudios de caso en ciudades asiáticas, utilizando un esquema que se basa principalmente en gestión de agua del subsuelo, ya que Wu y Tan (2012) determinaron el impacto en la atmósfera por la urbanización en la provincia de Shandong, China, así como la relación entre el cambio climático y el crecimiento urbano demostrando por medio de modelos matemáticos y diferentes escenarios que los cambios de cobertura y uso de suelo pueden causar inundaciones. De modo similar, Zhan et al. (2009) demostraron cómo la falta de vegetación en Beijing, debido a la expansión urbana, ha ocasionado un aumento en la temperatura, extensos flujos de calor y una menor evaporación. Resaltando la importancia de estos factores pues reducen la precipitación. Otras investigaciones ( $\mathrm{Li}$, Zhu, Sun, y Wang, 2010) estimaron el uso de suelo anual de Lian yungang, China, basado en imágenes satelitales para localizar y dimensionar la expansión urbana y la contaminación de cuerpos de agua; de igual forma, Seto, Güneralp y Hutyra (2012) estudió el cambio de suelo por la agricultura y el acelerado crecimiento de las ciudades relacionado con pérdida de biodiversidad. Por medio de modelación de la expansión urbana en ciudades de África como Kampala, capital de Uganda, y otras investigaciones que señalan los impactos negativos principalmente en los bosques de Guinea del este de África y el oeste de Sri Lanka (Seto et al, 2012).

Aun así, Torres et al. (2017) afirman que son pocos los trabajos existentes en relación con la medición o evaluación de la expansión urbana y sus impactos; siendo estos difíciles de comparar teniendo en cuenta que corresponden a adaptaciones a los casos de estudio que incluyen sistemas de información geográfica, listas de chequeos, matrices, modelos de simulación, etc. Se resalta la importancia de este estudio, pues busca predecir los impactos de la urbanización del área destinada a la reserva con relación a la oferta de bienes y servicios ecosistémicos.

\section{CONCLUSIONES}

La sustentabilidad es afectada en todas sus dimensiones al ocupar la RTvdH, desconociendo su funcionalidad ecosistémica y siendo el cambio del uso del suelo con tendencia a la urbanización el principal factor negativo.

El estudio permitió demostrar por medio de la modelación los procesos que pueden alterar el área de estudio en el cual se observan las tendencias y la relación directa entre el cambio del uso del suelo y el deterioro de los bienes y servicios ecosistémicos. Es así como se determina que para el año 2060 el cambio y deterioro en la oferta de bienes y servicios ambientales correspondería al 37\% de la reserva, presentando cambios en el $68.75 \%$ del área que oferta bienes y servicios ambientales de mantenerse las tasas actuales de cambio de uso del suelo, con tendencia a la urbanización. También se identificó la posible pérdida del aprovechamiento del agua sub-superficial como un bien y servicio de provisión y se calculó el crecimiento poblacional al interior de la reserva, la cual se estimó que se duplicaría para el periodo 2055-2056.

La simulación dinámica de los componentes ambientales de la RTvdH es el principal insumo para la generación de estrategias de gestión integrada de los sistemas socio-ecológicos, razón por la cual se debe consolidar la reserva, reduciendo la ocupación ilegal y el cambio de uso del suelo con tendencia a la urbanización.

\section{AGRADECIMIENTOS}

Los autores agradecen especialmente al Jardín Botánico de Bogotá José Celestino Mutis, el cual apoyó la investigación "Análisis de sustentabilidad ambiental por uso del suelo en la Reserva Forestal Productora Thomas van der Hammen Bogotá D.C.", con la entrega del estímulo a la investigación Thomas van der Hammen de la subdirección científica (resolución 422 del 20 de diciembre del 2016). 


\section{CONFLICTO DE INTERESES}

Los autores declaran no tener conflicto de intereses.

\section{CONTRIBUCIÓN POR AUTOR}

Los autores son los únicos responsables de la obra en todos los aspectos que condujeron a la elaboración de su publicación.

\section{REFERENCIAS BIBLIOGRÁFICAS}

\section{Academia Colombiana de Ciencias Físicas y Natura-} les (ACCEFY) (2011). Soporte de la declaración sobre la reserva forestal regional del norte -RFRNde Bogotá. Recuperado de http://www.reservathomasvanderhammen.co/index.php/documentos/ send/3-documentos-tecnicos-cientificos/4-declaracion-sobre-la-reserva-forestal-regional-del-norte-de-bogota

Acevedo, A. B. y Samper, G. (2003). Elementos de una política de vivienda popular. En G. Ardila (ed.), Territorio y sociedad: el caso del Plan de Ordenamiento Territorial de la ciudad de Bogotá (pp. 203206). Bogotá: Universidad Nacional de Colombia.

Álvarez Hincapié, C. F. (2010). Capital natural crítico y función de hábitat como aproximación a la complejidad ambiental. Revista Lasallista de Investigación, 7(2), 132-149.

Barroso, A. M., de Zárate, L. L. O. y Álvarez, A. M. A. (2010). Estudio de Caso. Retos para la sostenibilidad de las ciudades inteligentes. Madrid: Fundación José Ortega y Gasset-Gregorio Marañón, Goberna IUIOG.

Calvachi Zambrano, B. (2013). Lineamientos para el ordenamiento ambiental del borde sur de la ciudad de Bogotá y para consolidar un área de reserva ambiental. Mutis, 3(1), 86-131. DOI: https://doi. org/10.21789/22561498.842

Casas-Matiz, E. I. y Porras, X. A. C. (2017). Rehabilitación ecológica, Reserva Thomas van der Hammen.
Questionar: Investigación Específica, 5(1), 93-104.

DOI: https://doi.org/10.29097/23461098.103

Corporación Autónoma Regional de Cundinamarca (CAR) (2014a). Acuerdo 021 del 23 de septiembre del 2014, por medio del cual se adopta el Plan de Manejo Ambiental de la Reserva Forestal Regional Productora del Norte de Bogotá, D. C. "Thomas van der Hammen". Recuperado de http://www.car. gov.co/index.php?idcategoria $=48647$ \#

\section{Corporación Autónoma Regional de Cundinamarca} (CAR) (2014b). Objetos y objetivos de conservación, Plan de Manejo Ambiental de la Reserva Forestal Regional Productora del Norte de Bogotá D.C. "Thomas van der Hammen". Recuperado de http:// www.car.gov.co/index.php? idcategoria $=48654$

Cortés, M. G. O., García, J. I. U. y Díaz, G. P. (2015). Importancia económica y social de los servicios de los ecosistemas: una revisión de la agenda de investigación. Revista Global de Negocios, 3(2), 103-113.

Eberlein, R. L. y Peterson, D. W. (1992). Understanding models with Vensim ${ }^{\mathrm{TM}}$. European journal of operational research, 59(1), 216-219. DOI: https://doi. org/10.1016/0377-2217(92)90018-5

Eppink, F. V. (2004). Modelling biodiversity and land use: urban growth, agriculture and nature in a wetland area. Ecological Economics, 51(3), 201-216. DOI: https://doi.org/10.1016/j.ecolecon.2004.04.011

García, G. A., Hoz Sánchez, D. y Pfaffenbichler, P. (2008). Análisis de impactos en la tarifa de cobro por congestión en Madrid utilizando la dinámica de sistemas. Madrid: Universidad Politécnica de Madrid.

Gómez, D. E. y Jiménez, M. A. (2014). Generación de cartografía temática de la cobertura y uso del suelo en la Reserva Forestal Productora del Norte de Bogotá D.C. "Thomas van der Hammen" (RFPNBTVH), implementando la metodología Corine Land Cover (trabajo de pregrado, ingeniería en topografía). Bogotá: Universidad Distrital Francisco José de Caldas.

Gómez Rodríguez, D. M. (2017). Análisis de Sustentabilidad Ambiental por Uso del Suelo en la Reserva Forestal Productora, Thomas van der Hammen 
Bogotá D.C. (trabajo de posgrado, Maestría en Desarrollo y Gestión Ambiental). Universidad Distrital Francisco José de Caldas, Bogotá, Colombia.

González,Y.O. (2017). Conservación de vegetación para reducir riesgos hidrometereológicos en una metrópoli fronteriza. Estudios fronterizos, 18(35), 47-69. DOI: https://doi.org/10.21670/ref.2017.35.a03

Han, R. y Zhao, M. (2012). Evaluation on the Coordination of Economy and Environment with scarce Water Resources in Shandong Peninsula, China. Procedia Environmental Sciences, 13, 2236-2245.

Hincapié, C. F. (2014). Exergía en sistemas biológicos: aproximación holística para el estudio de ecosistemas y el manejo ambiental. Producción+ Limpia, $8(2), 106-127$.

Ibarra, D. W. (2013). Modelación de aspectos ambientales en la cadena de suministro del bioetanol. Ingeniería Industrial, 12(2), 79-93.

Kang, P. (2012). Water environmental carrying capacity assessment of an industrial park. Procedia Environmental Sciences, 13, 879-890. DOI: https://doi.org/10.1016/j.proenv.2012.01.082

Li, Y., Zhu, X., Sun, X. y Wang, F. (2010). Landscape effects of environmental impact on bay-area wetlands under rapid urban expansion and development policy: a case study of Lianyungang, China. Landscape and urban Planning, 94(3-4), 218-227.

Mas, J. F. y Flamenco, A. (2011). Modelación de los cambios de coberturas/uso del suelo en una región tropical de México. GeoTrópico, 5(1), 1-24.

Mazza, C. (2017). La ciudad desde más allá de la ciudad, 1930-1950. Hipótesis sobre algunas propuestas de equilibrio urbano-rural provenientes del agro. Registros. Revista de Investigación Histórica, 10, 4-23.

Montero, L. y García, J. (2017). Panorama multidimensional del desarrollo urbano en América Latina y el Caribe. Santiago de Chile: Naciones Unidas.

Mosquera, Y. M. (2015). Estrategias de gestión de riesgo fundamentada en la adaptación basada en ecosistemas (abe). Estudio de caso: cuenca Quebradanegra (Utica-Quebradanegra: Cundinamarca, Bogotá D.C.) (trabajo de posgrado, Maestría en Desarrollo y Gestión Ambiental). Universidad Distrital Francisco José de Caldas, Bogotá, Colombia.

Narváez, V. (2015). Modelo para la evaluación de la fiabilidad de sistemas dinámicos reparables de múltiples componentes (trabajo doctoral, Ingeniería Industrial). Universidad del Valle, Santiago de Cali, Colombia.

Quintero, J. C. V., Castro, C. A. C., Garcés, H. P. y Escobar, J. F. (2017). Procesos de geoprocesamiento en la espacialización de servicios ecosistémicos en áreas de interés local. Ingenierías USBmed, 8(1), 1928. DOI: https://doi.org/10.21500/20275846.2672

Rojas Bernal, C. L. (2016). Water infrastructure and social housing in Bogotá: an intersection between modern water management and social housing production. En: VIII Seminario Internacional de Investigación en Urbanismo. Barcelona: Departament d'Urbanisme i Ordenació del Territori. Universitat Politécnica de Catalunya.

Sánchez Díaz, C. (2016) Desarrollo y sostenibilidad de la reserva forestal Thomas van der Hammen: un acercamiento a la propuesta urbanística y expansiva de la actual administración del distrito capital. Red de Geografía Crítica de Raíz Latinoamericana, GeoRaízAL, 1(1), 1-19.

Seto, K. C., Güneralp, B. y Hutyra, L. R. (2012). Global forecasts of urban expansion to 2030 and direct impacts on biodiversity and carbon pools. Proceedings of the National Academy of Sciences, 109(40), 16083-16088. DOI: https://doi.org/10.1073/ pnas.1211658109

Torres, A. V., Martínez, S. A. y Nájera, R. M. S. (2017). Reflexión sobre enfoques metodológicos para la medición de la expansión urbana: el caso de la zona metropolitana de Toluca. En: B. V. Carrasco-Gallego (ed.), Megaproyectos urbanos productivos. Impactos socio-territoriales (pp. 105-117). Toluca: Universidad Autónoma del Estado de México.

Van der Hammen, T. y Andrade, G. (2003). Estructura ecológica principal de Colombia. Primera aproximación. Bogotá: Ministerio de Ambiente, Vivienda y Desarrollo Territorial, Instituto de Hidrología, Meteorología y Estudios Ambientales (Ideam). 
Ventana Systems (2015). Vensim PLE. Vensim ${ }^{\circledR}$ Software. Recuperado de http://vensim.com/download/

Verma, A. K., Srividya, A. y Karanki, D. R. (2010). Reliability and Safety Engineering. Londres: Springer Verlag. DOI: https://doi.org/10.1007/978-1-84996-232-2

Villanueva-Solis, J. R. M. (2013). Isla de calor urbana: modelación dinámica y evaluación de medidas de mitigación en ciudades de clima árido extremo. Información Tecnológica, 24(1), 15-24. DOI: https:// doi.org/10.4067/S0718-07642013000100003

Wei, S. Y. (2012). System dynamics simulation model for assessing socio-economic impacts of different levels of environmental flow allocation in the Weihe River Basin, China. European Journal of Operational Research, 221(1), 248-262. DOI: https://doi. org/10.1016/j.ejor.2012.03.014
Wu, P. y Tan, M. (2012). Challenges for sustainable urbanization: a case study of water shortage and water environment changes in Shandong, China. Procedia Environmental Sciences,13, 919-927.

Zhan, S. F., Zhang, X. C., Ma, C. y Chen, W. P. (2012). Dynamic modelling for ecological and economic sustainability in a rapid urbanizing region. Environmental Sciences, 13, 242-251. DOI: https://doi.org/10.1016/j.proenv.2012.01.023

Zhang, C. L., Chen, F., Miao, S. G., Li, Q. C., Xia, X. A. y Xuan, C. Y. (2009). Impacts of urban expansion and future green planting on summer precipitation in the Beijing metropolitan area. Journal of Geophysical Research: Atmospheres, 114, 1-26. DOI: https:// doi.org/10.1029/2008JD010328 
Anexo 1. Valores y formulas de la modelación dinámica

(01) Agropecuario $=986$

Units: hectáreas

986.60

(02) Agua recarga acuíferos=Precipitación promedio*suelo de recarga de acuíferos

Units: metros cúbicos/año

(03) agua superficial=3.88e+006-Cambio uso del suelo*Precipitación promedio

Units: metros cúbicos

388.9 ha de agua superficial

(04) Bienes y servicios Ambientales 0= INTEG (oferta 0/Cambio y Deterioro de BySA, 1295) Units: hectáreas/Year

(05) Bosques $=36$

Units: hectáreas

35.71

(06) Cambio uso del suelo $=267 / 13 * 46+$ Demanda de suelo

Units: hectáreas*Year

(07) Cambio y Deterioro de BySA=Bienes y servicios Ambientales 0-Cambio uso del suelo

Units: hectáreas/Year

(08) Coberturas artificiales $=\mathbf{1 6 5 . 6}$

Units: hectáreas

165.6

(09) Coberturas forestales=103Units: hectáreas 103.102

(10) Comercio y servicios $=7$

Units: hectareas7.43

(11) Conservación $=108$

Units: hectáreas

108.54

(12) Consumo=Reserva de agua superficial y acuíferos no confinados (Demanda agrícola de agua+ (Población*60))-1.80123e+006

Units: metros cúbicos/año

$5 \mathrm{~m} 3$ por 12 meses consumo por persona y $1.80123 \mathrm{e}+006$ agua de

Concesión al año supuesto de consumo 8 h 180 días al año
(13) culturales $0=$ Coberturas artificiales-Comercio $y$ servicios

Units: hectáreas/Year

(14) Demanda agrícola de agua=985.6*16000

Units: metros cúbicos/año

(15) Demanda de suelo $=0.0032 *$ Población

Units: hectáreas/personas

$32 \mathrm{~m} 2$ es el suelo que demanda una persona en Bogotá

(16) Dotacional $=95$

Units: hectáreas

95.36

(17) FINAL TIME $=2060$

Units: Year

The final time for the simulation.

(18) INITIAL TIME = 2001

Units: Year

The initial time for the simulation.

(19) migración=11.21/1000

Units: personas/año

(20) muertes=Población*tasa muertes

Units: personas/año

(21) nacimientos=Población*tasa nacimientos

Units: personas/año

(22) oferta $0=$ Culturales $0+$ Provision $0+$ Regulacion 0

Units: hectáreas/Year

(23) Población= INTEG (migración + nacimientosmuertes, 2095)

Units: personas/año

(24) Precipitación promedio $=982 / 1000$

Units: metros cuadrados/año

(25) Provisión 0=Agropecuario-Dotacional

Units: hectáreas*Year

(26) Regulación $0=$ Bosques+ Coberturas forestal+ Conservación

Units: hectáreas/Year

(27) Reserva de agua superficial y acuíferos no confinados=

INTEG ((Agua recarga acuíferos+ agua superficial)-Consumo, 0)

Units: metros cúbicos/año 
(28) Reservas de agua subterránea=4.12e+008-Consumo

Units: metros cúbicos

(29) SAVEPER = TIME STEP

Units: Year

The frequency with which output is stored.

(30) suelo de recarga de acuíferos $=40350$

Units: metros

4.035 hectáreas
(31) tasa muertes $=3.62 / 1000$

Units: 1/año

(32) tasa nacimientos $=16.51 / 1000$

Units: 1/año

(33) TIME STEP $=0.5$

Units: Year

The time step for the simulation. 